\title{
Trends in Resonance Energy Shifts and Decay Rates for Bose Condensates in a Harmonic Trap
}

\author{
Kunal Das and Thomas Bergeman \\ Department of Physics and Astronomy, SUNY, Stony Brook, NY 11794-3800 \\ and Institute for Theoretical Atomic and Molecular Physics, Harvard-Smithsonian Center for Astrophysics, 60 Garden Street, \\ Cambridge, MA 02138
}

(November 1, 2018)

\begin{abstract}
This is a study of quasi-discrete Bogoliubov quasi-particles in a spherically symmetric harmonic trap. We first evaluate analytically the aymptotic energy shifts of the high energy modes and find them to have $1 / \sqrt{n}$ dependence on the number of radial nodes, $n$, consistent with earlier semiclassical discussions. To address the question of the widths or decay rates, $\gamma$, we attempt to clarify previous discussions by deriving an implicit equation for $\gamma$ from an assumption of exponential decay. Numerically, we study the trends in the behavior of the widths as a function of temperature, energy, particle number and scattering lengths. In particular, we find that the width due to Landau decay rises rapidly at low $n$ and then declines, while the Beliaev decay rate rises slowly with $n$. As temperature $\rightarrow 0$, Beliaev decay reaches a constant $(>0$ for $n>0$ ), while the Landau decay rate goes to zero. The decay rate, $\gamma$, is approximately linear in the $s$-wave scattering length.
\end{abstract}

PACS numbers: 03.75.Fi, 05.30.Jp, 05.10.-a, 67.40.Db

\section{INTRODUCTION}

In many finite quantum systems, such as atoms, nuclei, and molecules, an extensive structure of excited states plays an important role in dynamical situations and for diagnostics. A homogeneous quantum fluid, such as liquid ${ }^{4} \mathrm{He}$, exhibits a few excitation modes whose energy as a function of wavenumber and temperature has been intensively studied 11. Harmonically trapped, dilute alkali atom Bose condensates have recently been found to have at least two prominent excitation modes [2 [5] and theoretically there is the full manifold of Bogoliubov quasi-particle modes. In calculating thermal averages, one can perform a sum over such discrete quasi-particle modes and obtain results comparable to the more commonly used semiclassical integral over momentum space [6]. This raises the issue of the width of higher-lying resonances, which is accentuated by recent observations of surface modes [7] and a chance observation of a longitudinal mode [8]. In other words, if experimental conditions are such that higher modes can be excited, under what circumstances are their widths narrow enough that they can be resolved? Could collective or single-particle excited states of Bose condensates become research tools as useful as Rydberg states of atoms?

We first focus on the energies of excited modes. Analytic results have been obtained [9 11] most notably from the hydrodynamic approximation, which involves various approximations to the kinetic energy. For highly excited states, these approximations break down. Without making the hydrodynamic approximation, Refs. [12 15] used Bogoliubov theory to numerically compute low-lying excitations. High-lying levels were calculated numerically by You and Walsworth 16. Semiclassical methods have enabled Csordás et al. 17, 18] to obtain precise expressions for the shifts (relative to bare harmonic oscillator states) of high lying excitations in a spherically symmet- ric trap. The more general case of cylindrical symmetry was found to lead to chaotic motion. In this paper, we present a very simple derivation of the lowest order approximation to the energy shifts, somewhat in the spirit of the quantum defect parameterization of excited Rydberg levels of atoms, and we also present numerical results from Bogoliubov-Popov theory.

Since the first experimental observations of resonance excitation and decay in harmonic traps [2 20], a number of theoretical approaches have been presented on the question of resonance width or decay [19 31]. Much of this effort has been directed toward the JILA data, although more recently Landau and also Beliaev damping of a "scissors" mode in a TOP trap has been observed at Oxford [32]. Arguably the most successful approach in explaining the JILA data has been kinetic theory with Gaussian Ansatz for the condensate and excited mode plus collision integrals for the condensate-noncondensate interaction [27,28]. It was concluded that at a certain temperature the observed mode changed character from out-of-phase to in-phase oscillation of the condensate and the thermal atoms, and with this interpretation the calculations matched the experimental data quite well. Good agreement has been offered also by calculations with a stochastic sampling of classical orbits corresponding to quasi-particle quantum states [21] and more recently with the dielectric formalism [29]. In the present work, we will not consider again this experimental data but instead address the question of the widths of highlying excitations in a spherically symmetric trap. Our approach makes use of accurate (numerical) quasi-particle modes, and is valid when the perturbation of the system is small. This approach has the advantage of making it possible to consider explicitly the decay channels for Landau and Beliaev decay processes. We will present numerical results for decay widths as a function of atom number, interaction strength and temperature. 
Expressions for Landau and Beliaev decay of resonance excitations of trapped Bose gases have been presented by Pitaevskii and Stringari 20, by Guillemas and Pitaevskii [30], and by Giorgini [25,26]. Effectively, we adopt the expressions given in these papers, but provide additional justification. Our discussion illuminates some of the many-body aspects and the analogy with Weisskopf-Wigner theory of radiative decay. One difficulty with previous expressions is that the terms in the sum over decay modes involved a delta function in energy, which, if interpreted exactly, will tend to be null for discrete quasi-particle states. By introducing the WeisskopfWigner Ansatz of an exponentially decaying initial state, we obtain decay terms with a Lorentz profile factor in place of the energy delta function.

In this paper, our numerical results are intended not primarily to establish precise values for the energy shifts and the damping widths, but instead to seek patterns and trends as a function of various parameters. We consider only a spherically symmetric trapping potential. An outline of the paper is as follows. In Sec. II, we review the basic equations of Bogoliubov-Popov theory. In Sec. III, we use time independent perturbation theory to establish analytically, within the Thomas-Fermi approximation, the asymptotic behaviour of the energy shifts. In Sec. IV.A we review the basic theory of exponential decay in time-dependent perturbation theory and then proceed to derive an implicit equation for the decay width. In Sec. IV.B, the lowest order interaction terms that lead to damping of an oscillating condensate are derived. Sec. IV.C uses these results in conjunction with the methods developed in Sec. IV.A to derive an expression for the decay rate of an oscillating condensate. Section V presents numerical methods and results of numerical computation for the width and its dependence on various physical parameters.

\section{HAMILTONIAN AND BOGOLIUBOV EQUATIONS}

The second-quantized grand-canonical Hamiltonian for a weakly interacting Bose gas in a spherical harmonic trap is

$$
\hat{K}=\int d \mathbf{r} \hat{\psi}^{\dagger}\left(H_{\mathrm{ho}}-\mu\right) \hat{\psi}+\frac{g}{2} \int d \mathbf{r} \hat{\psi}^{\dagger} \hat{\psi}^{\dagger} \hat{\psi} \hat{\psi},
$$

where $\mu$ is the chemical potential and

$$
H_{\mathrm{ho}}=\frac{-\hbar^{2} \nabla^{2}}{2 m}+\frac{1}{2} m \omega_{\mathrm{ho}}^{2} r^{2} ; \quad g=4 \pi \hbar^{2} a / m .
$$

with $a$ the s-wave scattering length and $\omega_{\text {ho }} / 2 \pi$ the harmonic frequency of the trap. The field operators are decomposed in the usual way into a macroscopic condensate wavefunction and a noncondensate operator

$$
\hat{\psi}(\mathbf{r}, t) \simeq\langle\hat{\psi}(\mathbf{r}, t)\rangle+\hat{\phi}(\mathbf{r}, t)=\Phi(\mathbf{r}, t)+\hat{\phi}(\mathbf{r}, t) .
$$

The temporal dependence of the condensate wavefunction is attributed entirely to a small amplitude oscillation (fluctuation) of the condensate about its equilibrium value $\Phi_{0}(\mathbf{r})$

$$
\Phi(\mathbf{r}, t)=\Phi_{0}(\mathbf{r})+\delta \hat{\Phi}(\mathbf{r}, t) .
$$

These substitutions are made and the resulting expressions linearized with respect to the fluctuations. Subsequently equilibrium mean fields $n_{T}(\mathbf{r})=\left\langle\hat{\phi}^{\dagger} \hat{\phi}\right\rangle$ and $m_{T}(\mathbf{r})=\langle\hat{\phi} \hat{\phi}\rangle$ are introduced to reduce the Hamiltonian to a quadratic form in the noncondensate operators. If $\Phi_{0}(\mathbf{r})$ satisfies the Gross-Pitaevskii equation

$$
\left[H_{\mathrm{ho}}-\mu+g\left(\left|\Phi_{0}\right|^{2}+2 n_{T}\right)\right] \Phi_{0}=0,
$$

the first order terms in $\hat{\phi}$ cancel out. The present work does not deal with the regime near the critical temperature, $T_{c}$, and thus the Popov approximation will be adequate and so terms in $m_{T}$ are neglected.

If the coupling between the fluctuations and the thermal operators are left out, the rest of the Hamiltonian is diagonalized by the Bogoliubov transformation:

$$
\begin{aligned}
& \hat{\phi}(\mathbf{r}, t)=\sum_{k}\left[u_{k}(\mathbf{r}) \hat{\alpha}_{k}(t)+v_{k}^{*}(\mathbf{r}) \hat{\alpha}_{k}^{\dagger}(t)\right] \\
& \hat{\phi}^{\dagger}(\mathbf{r}, t)=\sum_{k}\left[u_{k}^{*}(\mathbf{r}) \hat{\alpha}_{k}^{\dagger}(t)+v_{k}(\mathbf{r}) \hat{\alpha}_{k}(t)\right],
\end{aligned}
$$

provided that the functions $u_{k}(\mathbf{r})$ and $v_{k}(\mathbf{r})$ obey the Bogoliubov equations,

$$
\begin{array}{r}
{\left[H_{\mathrm{ho}}-\mu+2 g\left(\left|\Phi_{0}\right|^{2}+n_{T}\right)\right] u_{k}(\mathbf{r})+g\left(\left|\Phi_{0}\right|^{2}\right) v_{k}(\mathbf{r})} \\
=\epsilon_{k} u_{k}(\mathbf{r}) \\
-\left[H_{\mathrm{ho}}-\mu+2 g\left(\left|\Phi_{0}\right|^{2}+n_{T}\right)\right] v_{k}(\mathbf{r})-g\left(\left|\Phi_{0}\right|^{2}\right) u_{k}(\mathbf{r}) \\
=\epsilon_{k} v_{k}(\mathbf{r})
\end{array}
$$

In spherical symmetry, quantum numbers $n, \ell, m$ may be attached to the quasi-particle states $k$. In what follows, we will scale $\mathbf{r}$ by $r_{0}=\sqrt{\hbar / m \omega_{\mathrm{ho}}}$ and energies by $\hbar \omega_{\mathrm{ho}}$.

Numerical methods using the discrete variable representation (DVR) [34] have been applied [35,36] to solve the Gross-Pitaevskii and Bogoliubov equations. A thermal sum over discrete quasi-particle states is supplemented at high energies by an integral over a semiclassical representation.

\section{ASYMPTOTIC ENERGY SHIFTS}

We first consider the energy shifts of the collective and single particle modes of harmonically trapped condensate, relative to the energies of the harmonic oscillator modes. We are interested in the asymptotic behavior of the shifts as a function of the angular momentum, $l$, and the number of radial nodes $n$ of the excited modes. In this section we consider only zero temperature, which implies that almost all the atoms are in the condensate. 


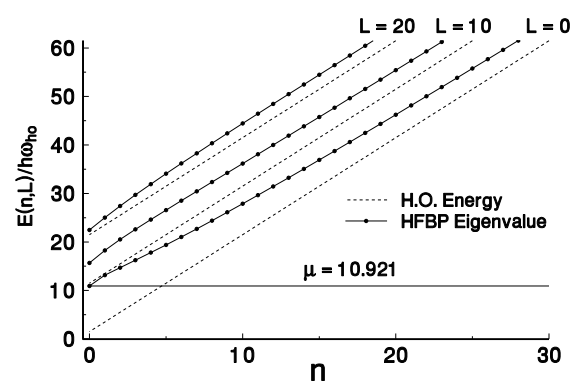

FIG. 1. Hartree-Fock Bogoliubov Popov (HFBP) and harmonic oscillator (HO) energies for $\ell=0,10$ and 20, relative to the trap minimum. The chemical potential is shown as a horizontal line. The scaled scattering length is $a / r_{0}=0.0072$ and number of atoms $N=20,000$.

By way of orientation, we first discuss overall trends of the energies $\epsilon_{n l}$, or more precisely, $E_{n l}=\epsilon_{n l}+\mu$. In Fig. 11, we have plotted numerically computed eigenvalues $E_{n l}$, for $T=0$ and for $l=0,10$ and 20 on an energy scale relative to the bottom of the harmonic well. On this scale, the condensate lies at an energy equal to the chemical potential $\mu$, shifted up from the harmonic zero point energy by the repulsive atom-atom interactions, and all excited states lie above the condensate. "Bare" harmonic oscillator energies $E_{n l}^{0}$ are shown for comparison. We will be interested in how the values $E_{n l}$ approach the $E_{n l}^{0}$, but first it is relevant to point out certain trends at low $n$.

For $\ell>0$, centrifugal distortion pushes the excited state wavefunction away from the condensate and thus reduces the shift for very small $n$. For larger $n$, the inner turning point moves inwards and the overlap with the condensate, and hence the energy shift, increases over a certain range of $n$. Then spreading of the excited state wavefunction to larger values of $r$ results in a decreasing shift. These effects are fully discussed in Ref. [18] in the semiclassical context.

A detailed and rigorous derivation of the energy shifts by WKB methods has been presented in [18] from a consideration of classical particle motion in the presence of the condensate. Trajectories that do not penetrate the condensate have excitation energies that are simply the harmonic oscillator energies,

$$
E_{n \ell}^{0}=(2 n+\ell+3 / 2) .
$$

For trajectories that do penetrate the condensate region, there is a shift:

$$
\delta_{n \ell}=E_{n \ell}-E_{n \ell}^{0}
$$

that is found to be 18

$$
\delta_{n \ell}=\frac{1}{3 \pi} \frac{\left[4 \mu(2 n+\ell+3 / 2-\mu)-(\ell-1 / 2)^{2}\right]^{3 / 2}}{[2 n+\ell+3 / 2-\mu]^{2}} .
$$

It is possible to obtain the $n \rightarrow \infty$ limit of the above expression by simple perturbation theory. For this purpose, we set $n_{T}(\mathbf{r})=0$ as appropriate for $T=0$. We define a $2 \times 2$ matrix Hamiltonian corresponding to the left hand sides of the coupled equations Eq. (7):

$$
\mathbf{H}_{\mathrm{Bog}}=\left(\begin{array}{cc}
H_{\mathrm{ho}}-\mu & 0 \\
0 & -H_{\mathrm{ho}}+\mu
\end{array}\right)+\left(\begin{array}{cc}
2 g\left|\Phi_{0}\right|^{2} & g\left|\Phi_{0}\right|^{2} \\
-g\left|\Phi_{0}\right|^{2} & -2 g\left|\Phi_{0}\right|^{2}
\end{array}\right) .
$$

The zeroth order eigenvector corresponding to eigenvalue $E_{n l}^{0}-\mu$ is $\left(\psi_{n l m}(\mathbf{r}), 0\right)^{T}$, where $\psi_{n l m}(\mathbf{r})$ is an eigenstate of the spherical harmonic oscillator Hamiltonian, $H_{\mathrm{ho}}$ :

$\psi_{n l m}(r, \theta, \phi)=\frac{\sqrt{2 n !}}{\sqrt{\left(n+l+\frac{1}{2}\right) !}} L_{n}^{l+\frac{1}{2}}\left(r^{2}\right) r^{l} e^{-\frac{1}{2} r^{2}} \frac{Y_{n l m}(\theta, \phi)}{\sqrt{4 \pi}}$.

We have used standard notation for the spherical harmonics $Y_{n l m}(\theta, \phi)$ and Laguerre polynomials $L_{n}^{l+\frac{1}{2}}\left(r^{2}\right)$. Considering the second matrix operator in Eq. (11), the lowest order energy shift is:

$$
\Delta E_{n l}=2 g\left\langle\left.\psi_{n l m}|| \Phi_{0}\right|^{2} \psi_{n l m}\right\rangle .
$$

An estimate of the behaviour of the shifts for large values of $n$ may be obtained from the Thomas-Fermi approximation for the ground-state density,

$$
\left|\Phi_{0}\right|^{2}=\frac{2 \mu-r^{2}}{2 g},
$$

and the asymptotic form of the Laguerre polynomials

$$
L_{n}^{l+\frac{1}{2}}\left(r^{2}\right) \simeq \frac{1}{\sqrt{\pi}} e^{\frac{1}{2} r^{2}} r^{-(l+1)} n^{\frac{l}{2}} \cos \left[2 \sqrt{n} r-(l+1) \frac{\pi}{2}\right] .
$$

Stirling's formula gives an approximate value

$$
\frac{n !}{\left(n+l+\frac{1}{2}\right) !} \approx \frac{1}{n^{l+\frac{1}{2}}} \frac{e^{l+\frac{1}{2}}}{\left[\left(1+\frac{l+\frac{1}{2}}{n}\right)^{n}\right]^{\left(1+\frac{l+1}{n}\right)}},
$$

which, for large values of $l$, can be further simplified to

$$
\frac{n !}{\left(n+l+\frac{1}{2}\right) !} \approx n^{-\left(l+\frac{1}{2}\right)} e^{-\frac{\left(l+\frac{1}{2}\right)(l+1)}{n}} .
$$

We thereby obtain the asymptotic first order shift in energy

$$
\begin{aligned}
\Delta E_{n l} & \simeq \frac{e^{-\frac{\left(l+\frac{1}{2}\right)(l+1)}{n}}}{\pi \sqrt{n}} \int_{0}^{\sqrt{2 \mu}} d r\left(2 \mu-r^{2}\right)\left[1+(-1)^{l} \cos (4 \sqrt{n} r)\right] \\
& \simeq \frac{2}{3 \pi} \sqrt{\frac{(2 \mu)^{3}}{n}} e^{-\frac{\left(l+\frac{1}{2}\right)(l+1)}{n}}
\end{aligned}
$$

to leading order in $n$. In the limit $\ell \ll n \rightarrow \infty$, the shift becomes 


$$
\delta_{n \ell}=\frac{2}{3 \pi} \sqrt{\frac{(2 \mu)^{3}}{n}},
$$

which agrees exactly with the asymptotic value of Eq. (10).

To compare the asymptotic value of the shift obtained numerically with the value obtained from Eq. (10), and also with values from Eqs. (16) through (18), we plot these respective values multiplied by $\sqrt{n}$ over a range of values of $n$ for $l=0,5$ and 10 in Fig. 2 (for $\left.N=50,000, a_{s c} / r_{0}=0.0073\right)$. For $\ell=0$ and also $\ell=1$, the simple perturbation theoretic results happen to be closer to the numerical results than Eq. (10), but for all high values of $\ell$ and increasingly so, the WKB result is much closer than the perturbation theoretic result with asymptotic Laguerre functions. However, the WKB result does tend to approach the asymptote more rapidly than the numerical results. It should also be noted that the use of the more accurate expression, Eq. (16) for Stirling's formula produces an improvement over the usual approximation.

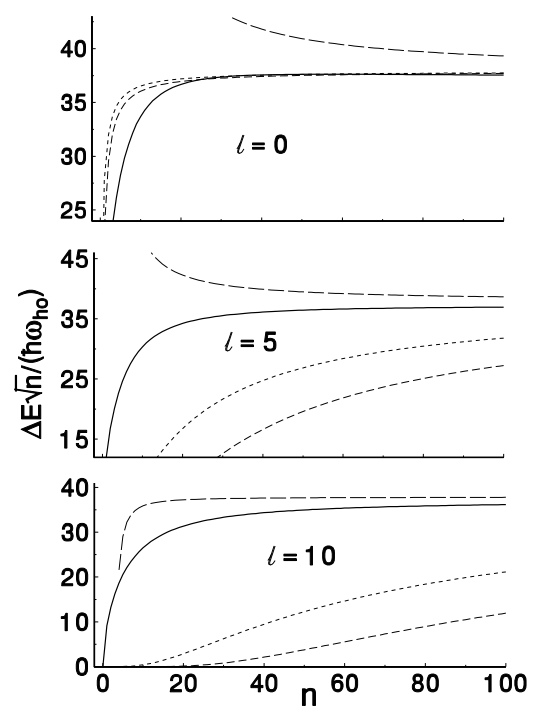

FIG. 2. Energy shifts $\Delta E$ multiplied by $\sqrt{n}$ are plotted versus $n$, the number of nodes in the radial wavefunction. The solid lines represent the energy shifts obtained from numerical solutions of the Bogoliubov equations (7). The long dashed lines above the solid lines represent the energy shifts calculated analytically from the WKB theory given in Eq. (10). The medium dashed lines show perturbation theory results of Eq. (17), while for the short dashed lines the more exact Stirling's approximation in Eq. (15) is inserted in Eq. (17). In each case, the scaled scattering length is $a / r_{0}=0.0072$ and number of atoms $N=50,000$.

We have also calculated first order energy shifts, Eq. (13) with exact harmonic oscillator wavefunctions rather than the asymptotic forms given above. The results are extremely close to the numerical results obtained with quasi-particle wavefunctions, from Eq. (7).

The fact that the asymptotic shift is independent of $\ell$ is demonstrated in Fig. 3 where we have plotted the energy shifts multiplied by $\sqrt{n}$ for $l=0,5,10$ and 20 . These shifts can be contrasted with the behaviour of Rydberg states of closed shell atoms, for which the asymptotic shift is $R \delta_{\ell} / n^{3}$, where the dipole polarizability is $\delta_{\ell}^{p o l} \simeq 3 \alpha_{d} / 4 \ell^{5}$, with $\alpha_{d}$ the dipolar polarizability. Thus for atomic Rydberg states, the asymptotic shift exhibits a sharp $\ell$ dependence that is lacking here. One other contrast with the theory of atomic Rydberg states is that the ion core can be considered effectively localized at the origin, while for excitations of a Bose condensate, a spatial integral, as in Eq. (18), is essential.

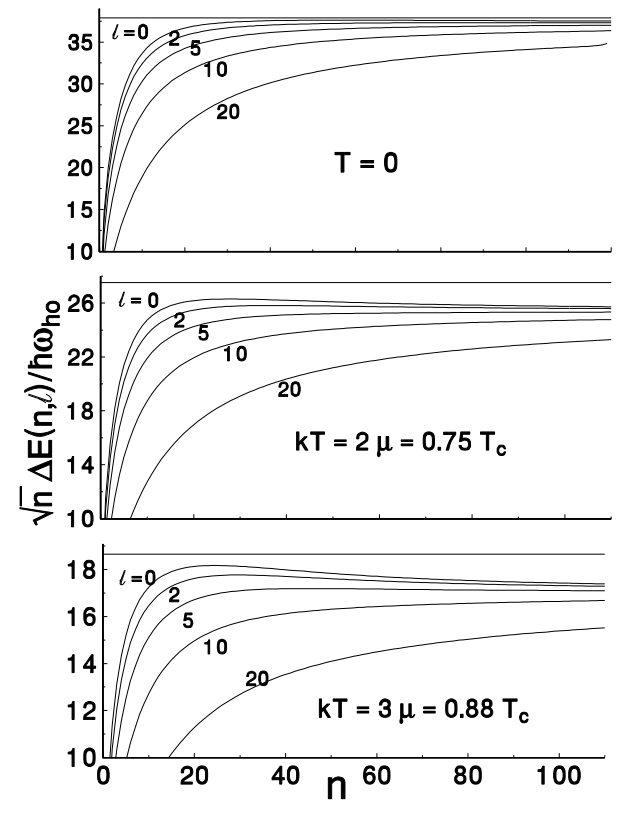

FIG. 3. Energy shifts $\Delta E=\operatorname{HFBP}(n, \ell)-\operatorname{HO}(n, \ell)$ multiplied by the $\sqrt{n}$ are plotted for different values of $\ell$, showing that the asymptotic shift is independent of $\ell$, and approximately equal to $2 \sqrt{(2 \mu)^{3}} / 3 \pi$. The scaled scattering length is $a / r_{0}=0.0072$ and number of atoms $N=50,000$.

In cylindrical symmetry, WKB wavefunctions exhibit chaotic behaviour [37], and the analysis cannot be carried out as for spherical symmetry. The asymptotic shift may be obtained from perturbation theory in a manner analogous to the above discussion, and results in the following expression:

$$
\Delta E_{n_{\rho}, n_{z}, m}=\frac{\mu^{2} e^{-\frac{m^{2}}{n_{\rho}}}}{8 \pi^{\frac{1}{2}} \sqrt{\omega_{\rho} n_{\rho} \omega_{z} n_{z}}}
$$

where $\omega_{\rho}$ and $\omega_{z}$ are trap frequencies, and $m$ is an exact 
quantum number. However, $n_{\rho}$ and $n_{z}$ are exact only when there is no condensate present.

\section{LANDAU AND BELIAEV DAMPING OF EXCITATIONS IN A TRAPPED BOSE GAS}

We now consider the damping of these collective and single particle excitations. An estimate of the width of the modes is essential to establish the feasibility of resolving high-lying excitations of a Bose condensate. The primary source of damping is the interaction of the collective excitation of the condensate with the noncondensate or thermal part. For high-lying excitations, the possibilities for both Landau and Beliaev decay increase with temperature, so it is interesting to obtain these rates explicitly.

A detailed exposition of the theory of Landau and Beliaev damping of quasi-particle excitations for Bose condensates has been presented by Giorgini [25]. The expressions for Landau damping were in agreement with expressions given earlier by Pitaevskii and Stringari [20], but the derivation was considerably more detailed. Giorgini's derivation is applicable to Bose atoms in harmonic trap potentials as well as to homogeneous Bose gases. Our discussion takes another point of view that results in expressions with a Lorentz profile. Consequently, all interacting levels within the linewidth of a resonant mode contribute to damping by Landau or Beliaev mechanisms. We note again that references [21] and [29] also present theories of Landau damping for trapped Bose atoms.

\section{A. Damping from Perturbation Theory}

We first review some of the methods of time-dependent perturbation theory 38,39 that will be used in our approach to the calculation of the widths. It will be convenient to work in the interaction picture, in which the time dependence of the operators and the states is given by

$$
\begin{gathered}
\hat{O}(t)=e^{i \hat{H}_{0} t / \hbar} \hat{O}_{S} e^{-i \hat{H}_{0} t / \hbar} \\
|\Psi(t)\rangle=e^{i \hat{H}_{0} t / \hbar}\left|\Psi_{S}(t)\right\rangle,
\end{gathered}
$$

where $\hat{O}_{S}$ signifies a time-independent operator in the Schrödinger picture. The Hamiltonian is $\hat{H}=\hat{H}_{0}+\hat{V}$. The differential equations that determine the time development are

$$
\begin{aligned}
i \hbar \frac{\partial}{\partial t}|\Psi(t)\rangle & =\hat{V}(t)|\Psi(t)\rangle \\
i \hbar \frac{\partial}{\partial t} \hat{O}(t) & =\left[\hat{O}(t), \hat{H}_{0}\right] .
\end{aligned}
$$

The time development of the state from time $t=0$ to a later time $t$ can be effected by means of a time-evolution operator $\hat{U}(t, 0)$ acting on the initial state

$$
|\Psi(t)\rangle=\hat{U}(t, 0)|\Psi(0)\rangle .
$$

From Eq. (23) it follows that the operator satisfies the differential equation

$$
i \hbar \frac{\partial}{\partial t} \hat{U}(t, 0)=\hat{V}(t) \hat{U}(t, 0)
$$

which can be integrated with the initial condition $\hat{U}(0,0)=1$ to give

$$
\hat{U}(t, 0)=1-\frac{i}{\hbar} \int_{0}^{t} d t^{\prime} \hat{V}\left(t^{\prime}\right) \hat{U}\left(t^{\prime}, 0\right) .
$$

The final state can be expanded in a basis of stationary eigenstates of $\hat{H}_{0}$

$$
|\Psi(t)\rangle=\sum_{n} a_{n}(t)\left|\Psi_{n}\right\rangle
$$

with coefficients

$$
\begin{aligned}
a_{n}(t) & =\left\langle\Psi_{n}|\hat{U}(t, 0)| \Psi(0)\right\rangle \\
& =\left\langle\Psi_{n} \mid \Psi(0)\right\rangle-\sum_{m} \frac{i}{\hbar} \int_{0}^{t} d t^{\prime}\left\langle\Psi_{n}\left|V\left(t^{\prime}\right)\right| \Psi_{m}\right\rangle a_{m}\left(t^{\prime}\right)
\end{aligned}
$$

At this point we make the simplifying assumption that the initial state is an eigenstate of $\hat{H}_{0},|\Psi(0)\rangle=\left|\Psi_{i}\right\rangle$. Moreover, if the time scales are short enough and there are a large number of possible final eigenstates, it can be assumed to a very good approximation that $a_{i}(t) \gg$ $a_{n \neq i}(t)$, so that

$$
a_{n \neq i}(t) \simeq-\frac{i}{\hbar} \int_{0}^{t} d t^{\prime}\left\langle\Psi_{n}\left|\hat{V}\left(t^{\prime}\right)\right| \Psi_{i}\right\rangle a_{i}\left(t^{\prime}\right)
$$

If the time scales are sufficiently short that changes in the initial state can be ignored, we can set $a_{i}(t) \approx 1$, and Eq. (29) can be solved for each $n \neq i$ to obtain:

$$
\left|a_{n}(t)\right|^{2}=2\left|\left\langle\Psi_{n}\left|V_{S}\right| \Psi_{i}\right\rangle\right|^{2}\left[\frac{1-\cos \left\{\left(\omega_{n}-\omega_{i}\right) t\right\}}{\left[\hbar\left(\omega_{n}-\omega_{i}\right)\right]^{2}}\right] .
$$

We have reverted to the Schrodinger picture in order to make the time-dependence explicit. $E_{n}=\hbar \omega_{n}$ is the energy associated with the $n$th eigenstate of the unperturbed Hamiltonian $H_{0}$. Then the usual procedure is to assume that $t \gg \hbar /\left(E_{n}-E_{i}\right)$, so that even though $t$ is still small compared with the inverse decay rate, one is justified in writing the quantity in brackets as a delta function. In this way, one obtains Fermi's Golden Rule:

$$
\Gamma_{n}=\frac{d}{d t}\left|a_{n}(t)\right|^{2}=\frac{2 \pi}{\hbar}\left|\left\langle\Psi_{n}\left|V_{S}\right| \Psi_{i}\right\rangle\right|^{2} \delta\left(E_{n}-E_{i}\right) .
$$

The sum of the partial widths, $\sum_{n} \Gamma_{n}=\Gamma$, gives the total decay rate at $t=0$.

This expression serves for the evaluation of transition probabilities in a wide range of physical problems. However, for application to decay rates, it requires that there 
be an intermediate range of times that are large compared with the oscillation periods but small compared with $\Gamma^{-1}$. For a typical radiative decay process, for example a $\mathrm{D}$ line of sodium with $\Gamma / \omega \approx 2 \times 10^{-8}$, this is no problem. For the resonance excitations of Bose condensates reported in Ref. [3], however, $\Gamma / \omega \approx 0.01$ to 0.1, which makes the $\delta$ function in Eq. (31) problematical. Hence it is appropriate to introduce the method of Weisskopf and Wigner [39,40], which removes the upper limit on the range of $t$ by dropping the assumption that $a_{i}(t) \approx 1$. This is done by introducing an exponential decay of the initial state:

$$
a_{i}(t)=e^{-\frac{1}{2} \Gamma t}
$$

Eq. (29) then becomes

$$
\begin{aligned}
a_{n}(t) & \simeq-\frac{i}{\hbar} \int_{0}^{t} d t^{\prime}\left\langle\Psi_{n}\left|\hat{V}_{S}\right| \Psi_{i}\right\rangle e^{i\left(\omega_{n}-\omega_{i}\right) t^{\prime}} e^{-\frac{1}{2} \Gamma t^{\prime}} \\
& =\left\langle\Psi_{n}\left|\hat{V}_{S}\right| \Psi_{i}\right\rangle \frac{1-e^{i\left(\omega_{n}-\omega_{i}\right) t} e^{-\frac{1}{2} \Gamma t}}{\hbar\left\{\left(\omega_{n}-\omega_{i}\right)+i \Gamma / 2\right\}} .
\end{aligned}
$$

Conservation of probability for a complete set of states for a closed system

$$
1-\left|a_{i}(t)\right|^{2}=\sum_{m \neq i}\left|a_{m}(t)\right|^{2}
$$

then gives

$$
\begin{aligned}
1-e^{-\Gamma t}= & \sum_{m \neq i} \frac{\left|\left\langle\Psi_{m}\left|\hat{V}_{S}\right| \Psi_{i}\right\rangle\right|^{2}}{\hbar^{2}\left\{\left(\omega_{m}-\omega_{i}\right)^{2}+(\Gamma / 2)^{2}\right\}} \\
& \times\left[1+e^{-\Gamma t}-2 e^{-\Gamma t / 2} \cos \left\{\left(\omega_{m}-\omega_{i}\right) t\right\}\right] .
\end{aligned}
$$

As it stands, this expression does not have any assumptions about the time-scales involved. We will take the long term limit, $t \rightarrow \infty$, in order to remove the explicit dependence on time

$$
\Gamma / 2=\sum_{m \neq i} \frac{\left|\left\langle\Psi_{m}\left|\hat{V}_{S}\right| \Psi_{i}\right\rangle\right|^{2}}{\hbar^{2}} \frac{\Gamma / 2}{\left(\omega_{m}-\omega_{i}\right)^{2}+(\Gamma / 2)^{2}}
$$

We have multiplied both sides by $\Gamma / 2$ to obtain an implicit equation for the width that can be solved iteratively with a knowledge of the stationary states and their energies. This is the general form we will use to obtain decay rates for excitations in a Bose gas.

\section{B. The Damping Interaction}

We now consider the operators and the basis states that are relevant for a Bose-condensed system. Since we will work within the grand canonical ensemble, $H_{0}$ in the previous section will be replaced by $K_{0}$, which is the part of the grand canonical Hamiltonian in Eq. (1) that is diagonalized by the Bogoliubov transformation, Eq. (6).
The eigenfunctions are the quasi-particle basis states as given in equations Eqs. (5) and (77). The quasi-particles themselves are considered to be in a thermal distribution determined by $K_{0}$, so that the relevant statistical operator is

$$
\frac{e^{-\beta \hat{K}_{0}}}{\operatorname{Tr} e^{-\beta \hat{K}_{0}}}
$$

Eigenstates of $K_{0}$ may be written in a Fock representation of quasi-particle occupation numbers $\left|n_{1}, n_{2}, \cdots\right\rangle$, and satisfy

$$
\hat{K}_{0}\left|n_{1}, n_{2}, \cdots\right\rangle=\left[\sum_{k} \epsilon_{k} n_{k}\right]\left|n_{1}, n_{2}, \cdots\right\rangle
$$

where $\epsilon_{k}=\hbar \omega_{k}$ is the energy and $n_{k}$ is the occupation number of the $k$ th quasi-particle mode. The creation and destruction operators for the quasi-particle modes are defined by

$$
\begin{aligned}
\hat{\alpha}_{k}\left|n_{1}, \cdots, n_{k}, \cdots\right\rangle & =\sqrt{n_{k}}\left|n_{1}, \cdots, n_{k}-1, \cdots\right\rangle \\
\hat{\alpha}_{k}^{\dagger}\left|n_{1}, \cdots, n_{k}, \cdots\right\rangle & =\sqrt{n_{k}+1}\left|n_{1}, \cdots, n_{k}+1, \cdots\right\rangle .
\end{aligned}
$$

They obey the standard Bose commutator relations and their time-dependence in the interaction picture follows from Eq. (23)

$$
\hat{\alpha}_{k}(t)=\hat{\alpha}_{k} e^{-i \omega_{k} t} \quad \hat{\alpha}_{k}^{\dagger}(t)=\hat{\alpha}_{k} e^{i \omega_{k} t} .
$$

In this representation, the matrix elements of the statistical operator are

$$
\begin{aligned}
& \frac{\left\langle n_{1}, n_{2}, \cdots\left|e^{-\beta \hat{K}_{0}}\right| n_{1}, n_{2}, \cdots\right\rangle}{\sum_{\left\{m_{1}\right\}}\left\langle m_{1}, m_{2}, \cdots\left|e^{-\beta \hat{K}_{0}}\right| m_{1}, m_{2}, \cdots\right\rangle} \\
= & \frac{\prod_{k} e^{-\beta \epsilon_{k} n_{k}}}{\prod_{k} \sum_{m_{k}} e^{-\beta \epsilon_{k} m_{k}}} .
\end{aligned}
$$

We next define the interaction that causes damping. We start with the interaction term in Eq. (1), separate the condensate wavefunction as done in Eq. (3), introduce the small amplitude fluctuation of Eq. (4) and linearize with respect to the fluctuation to get

$$
\begin{array}{r}
\frac{g}{2} \int d \mathbf{r} \hat{\psi}^{\dagger} \hat{\psi}^{\dagger} \hat{\psi} \hat{\psi} \approx g \int d \mathbf{r}\left\{\Phi_{0} \Phi_{0} \Phi_{0}\left(\delta \hat{\Phi}+\delta \hat{\Phi}^{\dagger}\right)\right. \\
+\Phi_{0} \Phi_{0}\left[\left(2 \delta \hat{\Phi}+\delta \hat{\Phi}^{\dagger}\right) \hat{\phi}^{\dagger}+\left(\delta \hat{\Phi}+2 \delta \hat{\Phi}^{\dagger}\right) \hat{\phi}\right] \\
+\Phi_{0}\left[\delta \hat{\Phi}^{\dagger} \phi \phi+2\left(\delta \hat{\Phi}+\delta \hat{\Phi}^{\dagger}\right) \hat{\phi}^{\dagger} \hat{\phi}+\delta \hat{\Phi}^{\dagger} \hat{\phi}^{\dagger} \hat{\phi}^{\dagger}\right] \\
\left.+\left(\delta \hat{\Phi}+\delta \hat{\Phi}^{\dagger}\right) \hat{\phi}^{\dagger} \phi \phi\right\}
\end{array}
$$

Here, and in subsequent expressions, for the sake of brevity, the spatial and temporal dependencies are not shown explicitly where they are obvious. In this paper, we assume that the condensate fluctuation mode is identical with one of the quasi-particle modes Eq. (6), which we designate by the subscript ' $o s$ ' : 


$$
\delta \hat{\Phi}(\mathbf{r}, t)=u_{o s}(\mathbf{r}) \hat{\alpha}_{o s}(t)+v_{o s}^{*}(\mathbf{r}) \hat{\alpha}_{o s}^{\dagger}(t) .
$$

We ignore the dynamics of the noncondensate, i.e. the noncondensate quasi-particles are assumed to be in static thermal equilibrium, with a population given by the Bose distribution function. As in [30], we regard the effect of the oscillation as an increase of the quasi-particle population of the mode labeled ' $o s$ ' from its value, $f_{o s}^{0}$ in a thermal distribution to a macroscopic value, which we call $n_{o s}$. Furthermore, we assume that the amplitude of the oscillation is small enough that the thermal equilibrium of the noncondensate particles is not affected.

Before making the quasi-particle substitutions, we note that the terms that are zeroth order in the noncondensate operators cannot contribute to the decay of the fluctuation due to conservation of energy. The first order terms will also be constrained by conservation of energy so that each quasi-particle removed from the condensate fluctuation will be compensated by an identical quasi-particle added to the noncondensate; this essentially leaves the number of quasi-particles in the condensate fluctuation unchanged. Thus the lowest order contribution to the decay of the condensate fluctuation comes from the terms bilinear in the noncondensate operators. Hence, the damping interaction is defined by

$$
\hat{V}(t)=g \int d \mathbf{r} \Phi_{0}\left[\delta \hat{\Phi}^{\dagger} \hat{\phi} \hat{\phi}+2\left(\delta \hat{\Phi}+\delta \hat{\Phi}^{\dagger}\right) \hat{\phi}^{\dagger} \hat{\phi}+\delta \hat{\Phi} \hat{\phi}^{\dagger} \hat{\phi}^{\dagger}\right]
$$

We transform to quasi-particles and retain all the terms that do not violate conservation of energy. These are grouped into terms (labeled $\hat{V}^{+}(t)$ ) that create a quasi-particle in the condensate fluctuation and those that annihilate one (labeled $\hat{V}^{-}(t)$ ):

$$
\hat{V}(t)=\hat{V}^{+}(t)+\hat{V}^{-}(t) .
$$

These terms include two processes: (i) Landau decay in which a quasi-particle from the condensate fluctuation interacts with a noncondensate quasi-particle to create a higher energy quasi-particle and (ii) Beliaev decay in which a condensate fluctuation quasi-particle decays into two lower energy quasi-particles. The two processes are schematically represented below

$$
\begin{array}{rlr}
E_{o s}+E_{j} \leftrightarrow E_{i} & & \text { Landau } \\
E_{o s} \leftrightarrow E_{i}+E_{j} . & & \text { Beliaev }
\end{array}
$$

$\hat{V}^{-}(t)$ correspond to these processes going from left to right while those included under $\hat{V}^{+}(t)$ proceed in the reverse direction.

At this point, we make the time-dependence of the quasi-particle operators explicit. Also, since we are considering a stationary condensate the quasi-particle wave functions $u_{k}$ and $v_{k}$ are taken to be real. After some algebra and rearrangements of the indices under the summation, we obtain

$$
\begin{aligned}
& \hat{V}^{+}(t)=\sum_{i, j}\left[L_{i j} e^{i \omega_{i j}^{-} t} \hat{\alpha}_{o s}^{\dagger} \hat{\alpha}_{j}^{\dagger} \hat{\alpha}_{i}+B_{i j} e^{i \omega_{i j}^{+} t} \hat{\alpha}_{o s}^{\dagger} \hat{\alpha}_{j} \hat{\alpha}_{i}\right] \\
& \hat{V}^{-}(t)=\sum_{i, j}\left[L_{i j} e^{-i \omega_{i j}^{-} t} \hat{\alpha}_{o s} \hat{\alpha}_{j} \hat{\alpha}_{i}^{\dagger}+B_{i j} e^{-i \omega_{i j}^{+} t} \hat{\alpha}_{o s} \hat{\alpha}_{j}^{\dagger} \hat{\alpha}_{i}^{\dagger}\right]
\end{aligned}
$$

with $\omega_{i j}^{ \pm}=\omega_{o s}-\left(\omega_{i} \pm \omega_{j}\right)$ and

$$
\begin{gathered}
L_{i j}=2 g \int d \mathbf{r} \Phi_{0}\left[u_{o s}\left(u_{i} v_{j}+u_{i} u_{j}+v_{i} v_{j}\right)+\right. \\
\left.v_{o s}\left(u_{i} v_{j}+u_{i} u_{j}+v_{i} v_{j}\right)\right] \\
B_{i j}=g \int d \mathbf{r} \Phi_{0}\left[u_{o s}\left(u_{i} u_{j}+v_{i} u_{j}+v_{j} u_{i}\right)\right. \\
\left.+v_{o s}\left(v_{j} u_{i}+v_{i} v_{j}+v_{i} u_{j}\right)\right] .
\end{gathered}
$$

\section{Calculation of the Width}

The expressions for the interaction derived in the previous section can now be applied to the methods developed in Sec. IV.A to obtain the decay rate. Since we consider finite temperatures, the system has to be described by a density matrix. We incorporate our considerations from the previous section into the definition of the density matrix. The condensate oscillation is treated as an increase in population of a particular mode, labeled with subscript ' $o s$ ', to a macroscopic value $n_{o s} \gg 1$. At the same time the enhanced population $n_{o s}$ is considered sufficiently small to leave the remaining modes well approximated by a thermal distribution. Thus we define the initial density matrix to be

$$
\rho(0)=\sum_{\substack{\left\{n_{k}\right\} \\ k \neq 0 s}} W^{\left\{n_{k}\right\}}\left|n_{1}, n_{2} . . n_{o s} . .\right\rangle\left\langle n_{1}, n_{2} . . n_{o s} . .\right| .
$$

It is characterized by a well-defined and fixed value of $n_{o s}$, for the condensate oscillation mode, whereas the other modes are thermally distributed over all possible values. This is reflected in the statistical weight

$$
W^{\left\{n_{k}\right\}}=\frac{\prod_{k \neq o s} e^{-\beta \epsilon_{k} n_{k}}}{\prod_{l \neq o s} \sum_{m_{l}} e^{-\beta \epsilon_{l} m_{l}}} .
$$

The time evolution of the diagonal matrix elements of the density matrix is given by

$$
\begin{aligned}
& \left\langle m_{1}, m_{2}, . .|\rho(t)| m_{1}, m_{2}, . .\right\rangle \\
= & \left\langle m_{1}, m_{2}, . .\left|\hat{U}(t, 0) \rho(0) \hat{U}^{\dagger}(0, t)\right| m_{1}, m_{2}, . .\right\rangle \\
= & \sum_{\substack{\left\{n_{k}\right\} \\
k \neq o s}} W^{\left\{n_{k}\right\}}\left|\left\langle m_{1}, m_{2}, . .|\hat{U}(t, 0)| n_{1}, n_{2} . . n_{o s} . .\right\rangle\right|^{2},
\end{aligned}
$$

where, $\hat{U}(t, 0)$ is the time evolution operator defined earlier. We will use it in the linear approximation

$$
\hat{U}(t, 0) \simeq \mathbf{1}-\frac{i}{\hbar} \int_{0}^{t} d t^{\prime} \hat{V}\left(t^{\prime}\right) .
$$


The states $\left|m_{1}, m_{2}, \cdots\right\rangle$ that evolve from the initial state can have arbitrary occupation numbers for all modes. For damping of the condensate oscillation mode, however, only states with $m_{o s} \neq n_{o s}$ are relevant, for which case

$$
\begin{aligned}
& \left\langle m_{1}, m_{2}, . .|\rho(t)| m_{1}, m_{2}, . .\right\rangle \\
\simeq & \sum_{\substack{\left\{n_{k}\right\} \\
k \neq o s}} \frac{W^{\left\{n_{k}\right\}}}{\hbar^{2}}\left|\int_{0}^{t} d t^{\prime}\left\langle m_{1}, m_{2}, . .\left|\hat{V}\left(t^{\prime}\right)\right| n_{1}, n_{2} . . n_{o s} . .\right\rangle\right|^{2}
\end{aligned}
$$

Now we introduce an Ansatz similar to the WeisskopfWigner hypothesis that the number of particles in the condensate oscillation mode decays exponentially, i.e.

$$
n_{o s}(t)=n_{o s} e^{-\Gamma t}
$$

This it Ansatz and the interaction derived in Eqs.(45)(48) allow us to evaluate the matrix elements in Eq. (53)

$$
\begin{gathered}
\left|\int_{0}^{t} d t^{\prime}\left\langle m_{1}, m_{2}, \cdots\left|\hat{V}^{+}\left(t^{\prime}\right)\right| n_{1}, n_{2} . . n_{o s} . .\right\rangle\right|^{2}= \\
\left.L_{i j}^{2}\left|\int_{0}^{t} d t^{\prime} e^{\left(i \omega_{i j}^{-}-\frac{1}{2} \Gamma\right) t^{\prime}}\right|\right|^{2}\left(n_{o s}+1\right)\left(n_{j}+1\right) n_{i} \mid \begin{array}{l}
m_{o s}=n_{o s}+1 \\
m_{j}=n_{j}+1 \\
m_{i}=n_{i}-1
\end{array} \\
+B_{i j}^{2}\left|\int_{0}^{t} d t^{\prime} e^{\left(i \omega_{i j}^{+}-\frac{1}{2} \Gamma\right) t^{\prime}}\right|^{2}\left(n_{o s}+1\right) n_{j} n_{i} \mid \begin{array}{c}
m_{o s}=n_{o s}+1 \\
m_{j}=n_{j}-1 \\
m_{i}=n_{i}-1
\end{array} \\
L_{i j}^{2}\left|\int_{0}^{t} d t^{\prime} e^{\left(i \omega_{i j}^{-}-\frac{1}{2} \Gamma\right) t^{\prime}}\right|^{2} n_{o s} n_{j}\left(n_{i}+1\right) \mid \begin{array}{l}
m_{o s}=n_{o s}-1 \\
m_{j}=n_{j}-1 \\
m_{i}=n_{i}+1
\end{array} \\
+B_{i j}^{2}\left|\int_{0}^{t} d t^{\prime} e^{\left(i \omega_{i j}^{+}-\frac{1}{2} \Gamma\right) t^{\prime}}\right|^{2} n_{o s}\left(n_{j}+1\right)\left(n_{i}+1\right) \mid \begin{array}{l}
m_{o s}=n_{o s}-1 \\
m_{j}=n_{j}+1 \\
m_{i}=n_{i}+1
\end{array}
\end{gathered}
$$

It is obvious that for any given set of occupation numbers $\left\{m_{1}, m_{2} ..\right\}$, only one of the above terms will differ from zero.

We add up all the non-zero elements that arise from Eq. (56) considering all possible values of $m_{1}, m_{2}, \cdots$, and subtract from them all the non-zero elements that arise from Eq. (55). This gives us the probability for the number of particles in the condensate oscillation mode decreasing by one after time ' $t$ ' when we start with $n_{\text {os }}$ particles in that mode. We use the macroscopic nature of the oscillation mode, which means $n_{o s}$ is large compared to 1 , to set $n_{o s} \simeq n_{o s}+1$ and obtain

$$
\begin{aligned}
& P\left(n_{o s}, t ;-1\right) \\
& =\sum_{\substack{\left\{n_{k}\right\} \\
k \neq o s}} W^{\left\{n_{k}\right\}} \frac{n_{o s}}{\hbar^{2}} \sum_{i, j}\left[L_{i j}^{2}\left|\int_{0}^{t} d t^{\prime} e^{\left(i \omega_{i j}^{-}-\frac{1}{2} \Gamma\right) t}\right|^{2}\left(n_{j}-n_{i}\right)\right. \\
& \left.+B_{i j}^{2}\left|\int_{0}^{t} d t^{\prime} e^{\left(i \omega_{i j}^{+}-\frac{1}{2} \Gamma\right) t}\right|^{2}\left(1+n_{j}+n_{i}\right)\right] .
\end{aligned}
$$

The sum over $\left\{n_{k}\right\}$ with the statistical weights essentially reproduces a thermal distribution of the modes of other than the condensate oscillation mode. In order to evaluate this, we consider a generic term,

$$
\begin{aligned}
\sum_{n_{1}, n_{2}, \cdots} \cdots & \frac{\prod_{k} e^{-\beta \epsilon_{k} n_{k}}}{\prod_{l} \sum_{m_{l}} e^{-\beta \epsilon_{l} m_{l}}} n_{j} \\
= & \frac{\prod_{k=1}^{j-1} \prod_{k=j+1}^{\infty}\left[\sum_{n_{k}} e^{-\beta \epsilon_{k} n_{k}}\right] \sum_{n_{j}} n_{j} e^{-\beta \epsilon_{j} n_{j}}}{\prod_{l} \sum_{m_{l}} e^{-\beta \epsilon_{l} m_{l}}} \\
= & \frac{\sum_{n_{j}} n_{j} e^{-\beta \epsilon_{j} n_{j}}}{\sum_{n_{j}} e^{-\beta \epsilon_{j} n_{j}}}=\frac{1}{e^{\beta \epsilon_{j}}-1}
\end{aligned}
$$

Thus the thermal sum has the effect of replacing

$$
n \rightarrow f_{j}=\left[e^{\beta \epsilon_{j}}-1\right]^{-1} .
$$

We divide by $n_{o s}$ to obtain the probability of the change per initial quasi-particle:

$$
\begin{aligned}
& P(1, t ;-1) \\
& =\frac{1}{\hbar^{2}} \sum_{i, j}\left[L_{i j}^{2}\left|\int_{0}^{t} d t^{\prime} e^{\left(i \omega_{i j}^{-}-\frac{1}{2} \Gamma\right) t}\right|^{2}\left(f_{j}-f_{i}\right)\right. \\
& \left.\quad+B_{i j}^{2}\left|\int_{0}^{t} d t^{\prime} e^{\left(i \omega_{i j}^{+}-\frac{1}{2} \Gamma\right) t}\right|^{2}\left(1+f_{j}+f_{i}\right)\right] .
\end{aligned}
$$

The time integrals can be treated in the same way as in our general discussion in Section IV A. In the long time limit i.e. $t \rightarrow \infty$, these integrals tend to

$$
\left|\int_{0}^{t} d t^{\prime} e^{\left(i \omega_{i j}^{ \pm}-\frac{1}{2} \Gamma\right) t^{\prime}}\right|^{2} \rightarrow \frac{1}{\left(\omega_{i j}^{ \pm}\right)^{2}+(\Gamma / 2)^{2}} .
$$

As for Eq. (36), in the limit $t \rightarrow \infty$, the condensate oscillation has damped out completely so that $P(1, t ;-1) \rightarrow$ 1 , and we can obtain an implicit equation for $\Gamma$. At this point, we introduce $\gamma=\Gamma / 2$, which corresponds to the width parameter $\gamma$ used in references [20,25,30]. Thus

$$
\begin{aligned}
\gamma=\frac{1}{\hbar^{2}} \sum_{i, j}\left[L_{i j}^{2}\right. & \frac{\gamma}{\left(\omega_{i j}^{-}\right)^{2}+\gamma^{2}}\left(f_{j}-f_{i}\right) \\
& \left.+B_{i j}^{2} \frac{\gamma}{\left(\omega_{i j}^{+}\right)^{2}+\gamma^{2}}\left(1+f_{j}+f_{i}\right)\right],
\end{aligned}
$$

where we have multiplied both sides by $\gamma=\Gamma / 2$ as in Eq. (36). In the numerical work, we will use this expression iteratively to calculate the width.

To deduce an approximate relation for the dependence of $\gamma$ on the coupling parameter, $g$, and hence on $a$, the scattering length, we consider just those $(i, j)$ values for which $\omega_{i j}^{ \pm}<\gamma$. For this subset of interactions, which include the largest contributions to the width, we have

$$
\gamma^{2} \approx \frac{1}{\hbar^{2}} \sum_{i, j}\left[L_{i j}^{2}\left(f_{j}-f_{i}\right)+B_{i j}^{2}\left(1+f_{j}+f_{i}\right)\right] .
$$


Since $L_{i j}$ and $B_{i j}$ from Eq. (48) are each linearly dependent on $g$, we conclude that $\gamma$ is approximately linearly dependent on $g$ and $a$.

\section{Comparisons and Caveats}

Previous expressions for the damping rate given in Refs. [20,25, 30] employed a delta function, as in Fermi's Golden Rule, Eq. (31). For example, for Landau damping, Eq. (41) of Ref. [25] is

$$
\gamma_{L}=4 \pi g^{2} \sum_{i j}\left|A_{i j}\right|^{2}\left(f_{i}^{0}-f_{j}^{0}\right) \delta\left(\hbar \omega_{0}+\epsilon_{i}-\epsilon_{j}\right)
$$

For a manifold of discrete states, this delta function would never be precisely satisfied. By assuming exponential decay, we have obtained a width expression involving a Lorentz function with a finite width, that can be solved iteratively for $\gamma$. Our discussion in Sec. IV.A also makes clear that the delta function is difficult to justify when the ratio $\gamma / \omega$ approaches values of 0.01 to 0.1 as is the case for excitations of Bose condensates. However, one can formally obtain a similar expression from Eq. (64) by replacing $\omega_{0}$, the unperturbed resonance frequency, with the perturbed $\omega$, which is then assumed to be distributed over a range of values characterized by a Lorentz profile with a width $\gamma$.

In the above derivation, we have explicitly calculated the decay of diagonal density matrix elements. However, if we consider $\hat{\alpha}_{o s}$ as a $c$-number, $\hat{\alpha}_{o s}=\hat{\alpha}_{o s}^{\dagger}=\sqrt{N_{o s}}$, and evaluate the time evolution of the squared amplitude of the condensate wavefunction [33]

$$
\begin{aligned}
& |\Phi(\mathbf{r}, t)|^{2}=\left|\Phi_{0}(\mathbf{r})+\delta \Phi(\mathbf{r}, t)\right|^{2}= \\
& \left|\Phi_{0}(\mathbf{r})\right|^{2}+2 \Phi_{0}(\mathbf{r}) \sqrt{N_{o s}}\left[u_{o s}(\mathbf{r})+v_{o s}(\mathbf{r})\right] \cos \left(\omega_{o s} t\right) e^{-\gamma t} \\
& +N_{o s}\left[u_{o s}(\mathbf{r})^{2}+v_{o s}(\mathbf{r})^{2}\right. \\
& \left.+2 u_{o s}(\mathbf{r}) v_{o s}(\mathbf{r}) \cos \left(2 \omega_{o s} t\right)\right] e^{-\Gamma t}
\end{aligned}
$$

we see that the "diagonal" term that decays as $e^{-\Gamma t}$ is second-order in the small amplitude fluctuations. The term linear in $\Phi_{0}(\mathbf{r})$ and linear in the fluctuations must be considered the primary observable oscillating function. This term oscillates with a frequency $\omega_{\text {os }}$ and decays as $e^{-\gamma t}$. It can be considered to arise from a coherent superposition of the condensate and the condensate fluctuation mode. For our derivation to be relevant to this term, one must assume that indeed $\gamma$ of Eq. (65) is equal to $\gamma$ of Eq. (62), i.e. that the "off-diagonal" element decays at one-half the rate of the "diagonal" element.

One can question whether the decay always follows a simple exponential law as we have assumed. For a more precise estimate of the time evolution of the resonance oscillation, numerical integration of coupled equations, including important off-diagonal density matrix elements such as the coherent term in Eq. (65), would be needed.
We note also that we have neglected the thermal atoms in the os mode, which will be significant at certain temperatures. An accurate calculation of the contribution of this mode to the damping process raises questions we have not resolved. However, in view of the additional constraint imposed by the Lorentz profile, we estimate that Landau damping by the os mode itself is not significant.

\section{NUMERICAL RESULTS FOR EXCITATION WIDTHS}

To obtain the energies $\epsilon=\hbar \omega$ and the quasi-particle wave-functions $u_{k}$ and the $v_{k}$, we solve self-consistently the Gross-Pitaevskii equation for the condensate and the Bogoliubov equations, as given in Eqs. (5) and (7). As stated above, here we accept the Popov approximation and neglect the anomalous density of the thermal particles, but always $n_{T}(\mathbf{r})$ is included. These equations are solved numerically using the discrete variable representation (DVR) [34,35], a method derived from Gaussian quadrature methods. The sums over discrete quasiparticle states are supplemented by local density approximation (LDA) expressions [41], which treat the highlying states by a semiclassical integral over momentum. More details of this part of the calculations can be found in 36 .

To compute the widths we put in a trial value of $\gamma$ on the right side of the implicit Eq. (36) and evaluate the new value of $\gamma$. The calculation is iterated until the input $\gamma$ is equal to the calculated $\gamma$ within a pre-defined tolerance. We made careful tests to ensure that the resultant widths are independent of various computational parameters. In particular, in evaluating the sum over states we include all states such that $\omega_{i j}^{ \pm}$fell within a specified range. For each mode studied, the range was increased until the width was insensitive (within the tolerance) to the increments. Also we verified that the width was insensitive to initial trial values. Most of our calculations were done for $\ell=0$ excitations. The set of perturbing levels ranged from $\ell=0$ up to $\ell_{\max }$, ranging from 20 to 80 , which was found sufficient to obtain a converged value of $\gamma$.

The above computational procedure is in principle more precise than the methods used in Ref. [30], which employ condensate and excited state wavefunctions computed at $T=0$ with populations appropriate to the stated $T$. Although therefore we do not expect exact agreement with these results, we made extensive comparisons to provide qualitative tests of our approach. For this purpose we used the same atom number values and scaled scattering length, $a / r_{0}=7.36 \times 10^{-3}$ as in [30]. For example, the temperature variation of the widths for $N=50,000$ and 150,000 from [30] and from our results is shown in Fig. 1 . 


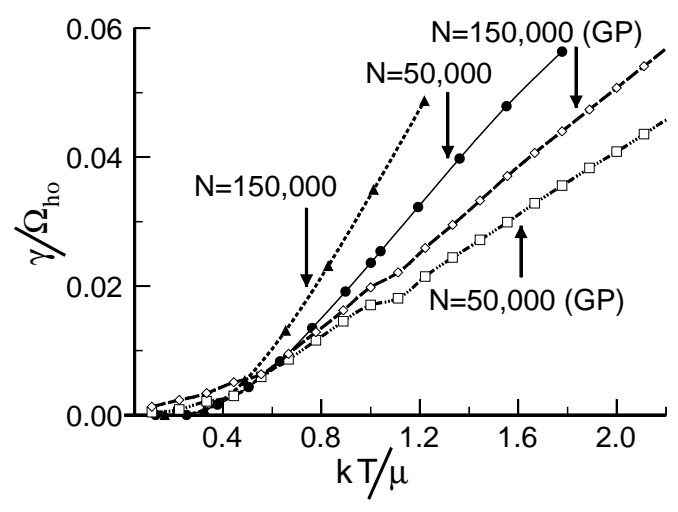

FIG. 4. The decay width, $\gamma$, for the lowest energy mode $(n=1)$, computed in this work as function of temperature is compared to the widths computed by Guilleumas and Pitaevskii (GP) in Ref. [30]. The width is scaled by the monopole resonance frequency $\Omega_{\mathrm{ho}} \simeq \sqrt{5} \omega_{\mathrm{ho}}$ and the temperature is scaled by the chemical potential, $\mu$, as in this reference.

From our numerical calculations for a spherically symmetric harmonic trap we observe the following trends:

(i) As in the data presented in [30], we find that the total width typically increases with temperature somewhat linearly, as shown in Fig. 5. For the higher resonances, non-zero values at $T=0$ reflect the Beliaev damping rate.

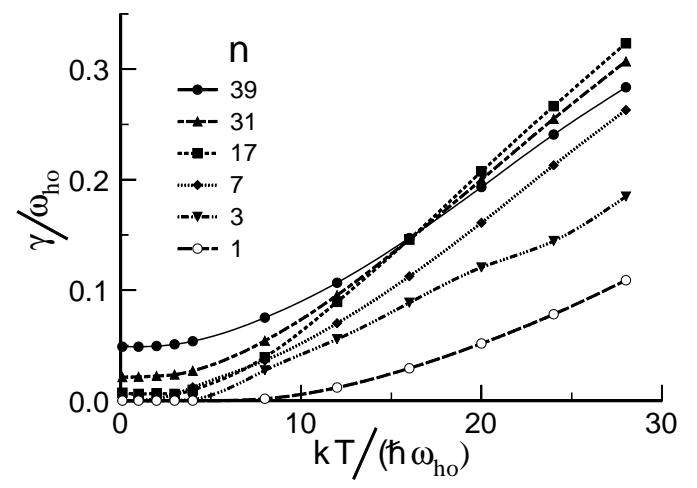

FIG. 5. The decay width $\gamma$ as function of scaled temperature, is shown for several condensate modes, for $\mathrm{N}=150,000$ and $a / r_{0}=0.00736$.

(ii) Figure 6 explains that the temperature increase of the width is due especially to the Landau contribution, while the Beliaev part increases much more slowly. This is plausible since the Landau process involves the interaction of a thermally populated quasi-particle mode with the oscillating mode in question, while the Beliaev process is simply a decay into two modes of lower energy. At higher temperature, of course there are more thermally populated modes available.

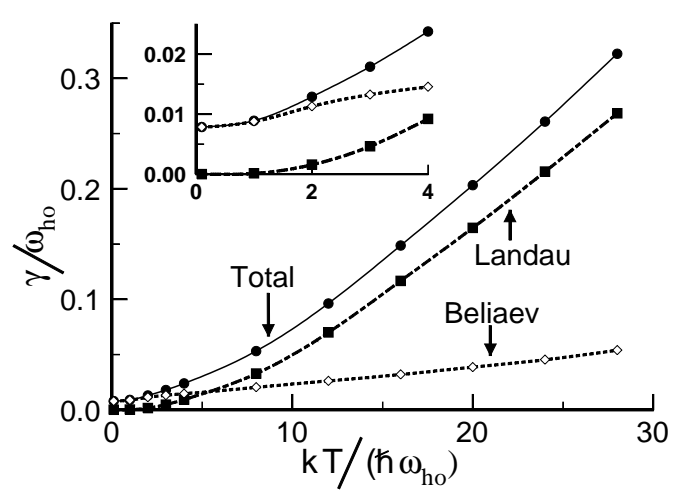

FIG. 6. The total decay width and the Landau and Beliaev contributions as function of scaled temperature $k T / \hbar \omega_{\text {ho }}$ are shown for the quasi-particle mode with $n=15, N=150,000$ and $a / r_{0}=0.00736$.

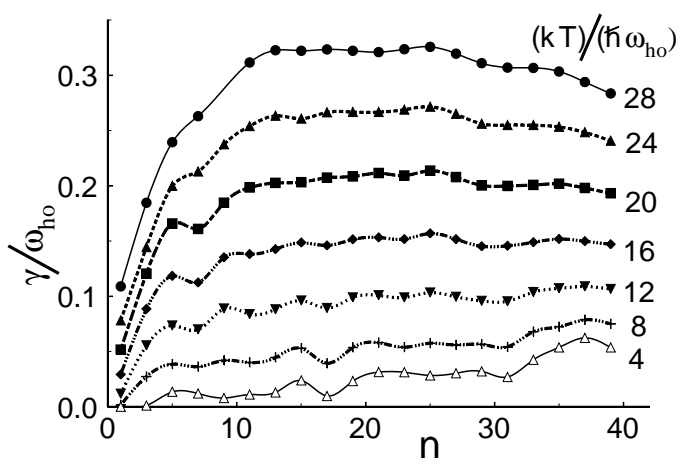

FIG. 7. The decay width $\gamma$ as function of the number of radial nodes, $n$, shown for a wide range of scaled temperatures, for $N=150,000$ and $a / r_{0}=0.00736$.

(iii) The total width rises sharply for low $n$, the radial quantum number, and then reaches a nearly flat plateau at a value of $n$ roughly proportional to $T$, as shown in Fig. 7. We have correlated this effect with the factor $f_{j}^{0}-f_{i}^{0}$ in Eq. (62). For the $(j, i)$ values that contribute most to $\gamma$, at higher temperatures, $f_{j}^{0}$ is more nearly equal to $f_{i}^{0}$. The temperature scale at which this occurs is set approximately by $\left(E_{i}-E_{j}\right) / k$. At higher $n$ values, $f_{i}^{0}$ is negligible compared with $f_{j}^{0}$, so the width as a function 
of $n$ flattens out. Note in this figure that for low values of the temperature, the width is small compared with $\hbar \omega_{h o}$ even for high values of $n$.

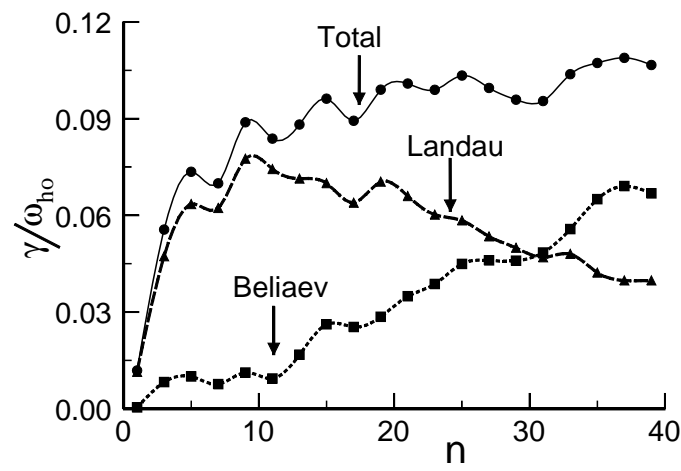

FIG. 8. The total decay width and the Landau and Beliaev contributions as function of the number of radial nodes $n$ of the condensate fluctuation are shown for scaled temperature $k T / \hbar \omega_{\text {ho }}=12, N=150,000$ and $a / r_{0}=0.00736$.

(iv) Figure 8, again breaks this trend down into Landau and Beliaev components for one particular case. The apparent plateau occurs because of a near balance between a decrease of the Landau rate and an increase in the Beliaev rate. Since for higher energy, there are more final states available, one does expect an increase in the Beliaev rate, but it is surprising that the increase is as gradual as it is. Also, the $n$ dependence of the Landau rate is rather unexpected. We attribute the decrease at large $n$ to the decrease of the overlap integrals in Eq. 48, since the excited state wavefunction will be increasingly extended in space.

(v) The total width increases with scattering length in approximately linear fashion, as shown for one set of conditions in Fig. 9. This is consistent with Eq. 63.) A larger scattering length implies stronger interaction among the particles and hence more rapid damping.

(vi) In Fig. 10, we have plotted widths for three different particle numbers. With fewer particles, the condensate is smaller and also the quasi-particle wavefunctions are closer to bare harmonic oscillator functions. For both reasons, the integrals in Eqs. (48) yield smaller values for the coupling parameters.

(vii) Especially for relatively low temperature and smaller values of the scaled scattering length, the widths are relatively small compared to the energy level separation, even at high values of energy or $n$. Unfortunately, if one attempts to excite high energy levels, typically many different $\ell$ values would be excited and would overlap.
However, if means can be found to achieve a perfectly spherically symmetric harmonic trap and then to excite a singlet $\ell$ manifold, our conclusion is that the highly excited levels would be resolvable in this ideal case.

(viii) Although the above results were entirely for the case $\ell=0$, we have performed calculations for $\ell$ up to 6 . For the cases studied, have found only a slight variation of the width with $\ell$.

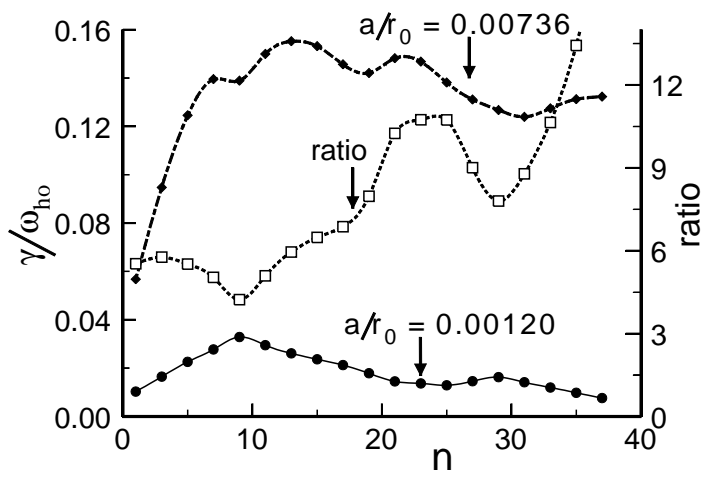

FIG. 9. The decay width $\gamma$ as function of number of radial nodes, $n$, of the condensate fluctuation, shown for two different values of the scaled scattering length $a / r_{0}$. The data are for $N=50,000$, and $k T / \hbar \omega_{\text {ho }}=16$. Also shown is the ratio of the widths (right axis), which may be compared with the value of 6.13 for the ratio of the scattering lengths.

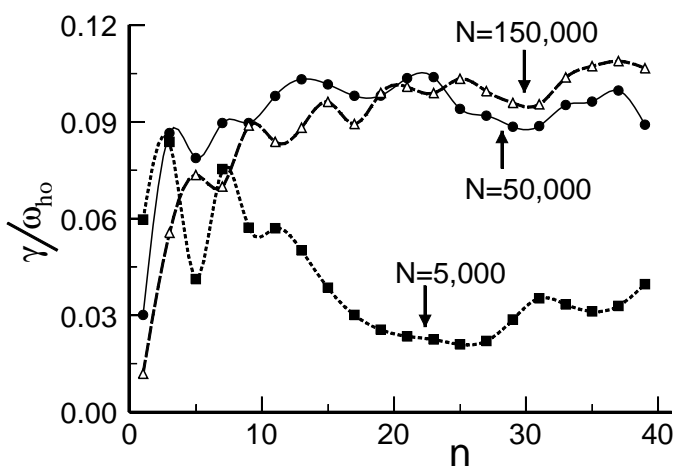

FIG. 10. The decay width, $\gamma$, as function of number of radial nodes, $n$, of the condensate mode, is shown for three different values of the number of particles, $N$. The data are for scaled scattering length $a / r_{0}=0.00736$ and $k T / \hbar \omega_{\text {ho }}=12$. 


\section{DISCUSSION AND CONCLUSIONS}

A key question motivating this study has been the extent of validity of the quasi-particle representation for trapped Bose atoms. In light of recent kinetic theory results [27 28], it may be that the quasi-particle representation is not essential for considering resonance decay. The appealing but complex picture of Landau and Beliaev decay processes may not be necessary. However, the Gross-Pitaevskii equation and the Bogoliubov quasiparticle approach to Bose condensates has been shown to be a powerful method for modeling thermal equilibrium situations. Also in view of past successes [21, 15], it seems worthy of further study as well as application to more highly excited resonances as we have done here. The dielectric formalism [29] employs quasi-particle basis states, but goes beyond the present approximations. We are currently analyzing these other approaches to make comparisons.

It is useful to distinguish between discrete quasiparticle modes, characterized by eigenvalues $\epsilon_{k}$ and eigenfunctions $u_{k}(\mathbf{r})$ and $v_{k}(\mathbf{r})$ from Eq. (7), and semiclassical quasi-particle functions, $u(\mathbf{p}, \mathbf{r}), v(\mathbf{p}, \mathbf{r})$ as used in [6]. The widths that we have computed for resonantly excited states may be presumed to apply also to the thermal quasi-particle states, which must thus be considered quasi-discrete quasi-particle (QDQP) states. When the widths of the QDQP states are comparable to their spacing, one can conclude that discrete basis states are not relevant, and a local semiclassical representation will suffice. Spherical symmetry presents a special case because the single-particle excitations at sufficiently high energy within a given $\ell$ manifold have a spacing of $2 \hbar \omega_{h o}$. We do find that for $N=150,000$ for example, for $T<12 \hbar \omega_{h o} / k$, typical widths are $<0.10 \hbar \omega_{h o}$, even for high-lying states with $n$ up to at least 30. Going from spherical to cylindrical symmetry, the spacing between levels within a given $m$ manifold decreases with energy, and thus semiclassical quasi-particle functions are more likely to be accurate. Consistent with this, we have observed that the semiclassical quasi-particle functions become more reliable at lower energies in cylindrical than in spherical symmetry for calculating thermal sums over quasi-particle states [36,42].

Very recently, a harmonic trap close to spherical symmetry has been produced [43], and we are informed [44] that axial and transverse frequencies can be made equal within about 1\%. Although we have found above that resonances within a given $\ell$ manifold are under certain circumstances resolvable, to our knowledge no one has yet devised an excitation method that would be $\ell$ selective, as are electric dipole excitations of atomic Rydberg levels. One can hope that such techniques for this purpose will be developed. You and Walsworth 16] have suggested that time-varying magnetic fields be configured so as to excite specific modes. With the help of focused laser beams, the MIT group has observed reso- nances with $n_{z}$ as high as 8 in a cigar-shaped trap, and surface modes [8] were excited by a sharply focused perturbing laser beam, moved rapidly around the transverse surface of the "cigar" trap. The technique of stimulated Bragg scattering [45 47] offers the capability to select the excitation energy and wavenumber, but is not $\ell$ selective in its current form. Possibly by special tailoring of the laser beams, one could achieve a nearly spherically symmetric excitation function.

Our review of the underlying theory of Bose condensate damping processes has exposed some interesting contrasts with the Weisskopf-Wigner theory of radiative damping. The decay rates of Bose condensate excitations are typically not a small fraction of the frequency, which makes the usual delta function limit problematical. Furthermore, the decay of interest is very likely the "off-diagonal" density matrix element between the condensate and the excited mode rather than the excitation intensity. In the language of NMR, we have calculated here $T_{1}$, but $T_{2}$ may be what is observed. To model these various circumstances in a more accurate manner, it may be necessary to explicitly integrate coupled timeevolution equations for various diagonal and off-diagonal elements. Also, the assumption of simple exponential decay may not always be valid. Modulated decays have been reported in calculations with an assumed 1D geometry [48] and also from Monte Carlo procedures [49]. As is well known, a system composed only of discrete frequencies will exhibit recurrences rather than damping. This conclusion may not apply if the number of interacting levels is effectively infinite, but at sufficiently low temperature, there may not be effectively an infinite number of accessible quasi-particle levels. Again, more detailed modeling of the time evolution may be needed.

In summary, we have presented numerical and perturbation theory results for excitation frequencies of Bose particles in a spherically symmetric harmonic trap, and compared with previously derived expressions. The energies approach the bare harmonic oscillator values asymptotically as $(4 / 3 \pi) \mu^{3 / 2} / \sqrt{2 / n}$ independent of $\ell$ (for $n>>$ $\ell$ ), where $n$ is the number of nodes in the radial equation. The asymptotic regime is reached more slowly for higher $\ell$. Secondly, we have presented a derivation of the excitation widths in agreement with previous discussions, but based from the beginning on an assumption of exponential decay, with an ensemble of discrete quasiparticle states, hence yielding a Lorentzian width expression rather than a delta function. Thirdly, we have presented numerical results for the widths over a range of parameter values. They showing that the Landau process rises more rapidly with temperature, while the Beliaev process rises more rapidly with $n$, except for very low $n$. Also, compared with the frequency separation of $2 \hbar \omega$ within an $\ell$ manifold, the widths are often small, especially for low temperatures and smaller values of the scaled scattering length.

This work was supported by ONR and NSF. We gratefully acknowledge valuable discussions with D. L. Feder, 
N. L. Balazs, B. I. Schneider, H. J. Metcalf, A. Griffin, A. Kuklov and R. Walser.

[1] A. Griffin, Excitations in Bose-Condensed Liquid, (Cambridge, 1993).

[2] D. S. Jin, J. R. Ensher, M. R. Matthews, C. E. Wieman, and E. A. Cornell, Phys. Rev. Lett. 77, 420 (1996).

[3] D. S. Jin, M. R. Matthews, J. R. Ensher, C. E. Wieman, and E. A. Cornell, Phys. Rev. Lett. 78, 764 (1997).

[4] M.-O. Mewes, M. Andrews, N. van Druten, D. Kurn, D. Durfee, C. Townswend, and W. Ketterle, Phys. Rev. Lett. 77, 988 (1996).

[5] D. Stamper-Kurn, H.-J. Miesner, S. Inouye, M. Andrews, and W. Ketterle, Phys. Rev. Lett. 81, 500 (1998).

[6] S. Giorgini, L. P. Pitaevskii, and S. Stringari, J. Low Temp. Phys. 109, 309 (1997).

[7] R. Onofrio, D. Durfee, C. Raman, M. Köhl, C. Kuklewicz, and W. Ketterle, Phys. Rev. Lett. 84, 810 (2000).

[8] W. Ketterle, D. Durfee and D. Stamper-Kurn, in BoseEinstein Condensation in Atomic Gases, Proceedings of the International School of Physics Enrico Fermi, Course $C X L$, ed. M. Inguscio, S. Stringari, and C. E. Wieman, (IOS Press, Amsterdam, 1999).

[9] S. Stringari, Phys. Rev. Lett. 77, 2360 (1996).

[10] W.-C. Wu and A. Griffin, Phys. Rev. A 54, 4204 (1996).

[11] P. Öhberg, E. Surkov, I. Tittonen, S. Stenholm, M. Wilkens, and G. Shlyapnikov, Phys. Rev. A 56, R3346 (1997).

[12] D. A. W. Hutchinson, E. Zaremba and A. Griffin, Phys. Rev. Lett. 78, 1842 (1997).

[13] L. You, W. Hoston and M. Lewenstein, Phys. Rev. A 55, R1581 (1997).

[14] D. Hutchinson and E. Zaremba, Phys. Rev. A 57, 1280 (1998).

[15] D. Hutchinson, R. Dodd, and K. Burnett, Phys. Rev. Lett. 81, 2198 (1998).

[16] L. You, R. Walsworth and W. Hoston, Optics Express 1, 293 (1997).

[17] A. Csordás, R. Graham, and P. Szépfalusy, Phys. Rev. A 54, R2543 (1996).

[18] A. Csordás, R. Graham, and P. Szépfalusy, Phys. Rev. A 56, 5179 (1997); Ibid. 57, 4669 (1998).

[19] W. Liu, Phys.Rev.Lett. 79,4056 (1997)

[20] L.P. Pitaevskii and S. Stringari, Phys. Lett. A 235,398 (1997).

[21] P. O. Fedichev, G. V. Shlyapnikov and J. T. M. Walraven, Phys. Rev. Lett. 80, 2269(1998)

[22] P. O. Fedichev and G. V. Shlyapnikov, Phys. Rev. A 58, 3146 (1998)

[23] G. M. Kavoulakis, C. J. Pethick and H. Smith Phys. Rev. A 61, 063605 (1998)

[24] G. Bene and P. Szépfalusy, Phys. Rev. A 58,3391 (1998)

[25] S. Giorgini, Phys. Rev. A 57,2949 (1998)

[26] S. Giorgini, cond-mat/9911377

[27] M. J. Bijlsma and H. T. C. Stoof, Phys. Rev. A 60, 3973
(1999).

[28] U. A. Khawaja and H. T. C. Stoof, cond-mat/0003517

[29] J. Reidl, A. Csordás, R. Graham and P. Szépfalusy, Phys.Rev. A 61,043606 (2000)

[30] M. Guilleumas and L. P. Pitaevskii, Phys. Rev.A 61,013602 (2000).

[31] J. E. Williams and A. Griffin, cond-mat/0003481

[32] G. Hodby, O. M. Maragò, G. Hechenblackner and C. J. Foot, cond-mat/0010157(Oct. 2000).

[33] M. Edwards, R. J. Dodd, C. W. Clark, and K. Burnett, J. Res. Natl. Inst. Stand. Tech. 101, 553 (1996).

[34] D. Baye and P.-H. Heenan, J. Phys. A. 19, 2041 (1986).

[35] B. I. Schneider and D. L. Feder, Phys. Rev. A 59, 2232 (1999).

[36] T. Bergeman, D. Feder, N. L. Balazs, and B. Schneider, Phys. Rev. A 58, 063605 (2000).

[37] M. Fleisser, A. Csordás, R. Graham, and P. Szépfalusy, Phys. Rev. A. 56, 4879 (1997).

[38] A. Fetter and J. D. Walecka, Quantum Theory of Many Particle Systems (McGraw-Hill, New York, 1971)

[39] W. Heitler, The Quantum Theory of Radiation, (Oxford, London, 1954).

[40] V. Weisskopf and E. Wigner, Z. Phys. 63, 54 (1930); Ibid. 65, 18 (1930).

[41] J. Reidl, A. Csordás, R. Graham, and P. Szépfalusy, Phys. Rev. A 59, 3816 (1999).

[42] T. Bergeman, unpublished work.

[43] B. P. Anderson, P. C. Haljan, C. A. Regal, D. L. Feder, L. A. Collins, C. W. Clark, and E. A. Cornell, condmat/0012444 (Dec. 2000).

[44] E. Cornell, private communication (November, 2000).

[45] J. Stenger, S. Inouye, A. Chikkatur, D. Stamper-Kurn, D. Pritchard, and W. Ketterle, Phys. Rev. Lett. 82, 4569 (1999).

[46] D. Stamper-Kurn, A. Chikkatur, A. Görlitz, S. Inouye, S. Gupta, D. Pritchard, and W. Ketterle, Phys. Rev. Lett. 83, 2876 (1999).

[47] F. Zambelli, L. Pitaevskii, D. Stamper-Kurn, and S. Stringari, cond-mat/9912089 (Dec. 1999).

[48] S. Choi, S. A. Morgan and K. Burnett, Phys. Rev. A 57, 4057 (1998).

[49] B. Jackson and C. S. Adams, cond-mat/0009468 (Sept. 2000). 